\title{
Histological phenotype is correlated with the wall-invasion pattern of gall- bladder adenocarcinoma
}

\author{
Takahito ToвA $^{1,2}$, Hiroshi KIJIMA ${ }^{2}$, Kenichi HaKamada ${ }^{3}$, and Yoshinori Igarashi ${ }^{1}$ \\ ${ }^{1}$ Division of Gastroenterology and Hepatology, Department of Internal Medicine, Toho University Omori Medical Center, Tokyo 143- \\ 8541, Japan; Departments of ${ }^{2}$ Pathology and Bioscience, and ${ }^{3}$ Gastroenterogical Surgery, Hirosaki University Graduate School of Med- \\ icine, Hirosaki 036-8562, Japan
}

(Received 3 June 2014; and accepted 23 July 2014)

\begin{abstract}
Gallbladder carcinoma (GBC) is one of the most aggressive malignancies, and frequently shows vascular invasion and lymph node metastasis. Our previous study has classified the wall-invasion pattern of GBC into two groups, i.e., infiltrative growth type (IG type) and destructive growth type (DG type). The DG type was significantly associated with poor clinical outcome. In this study, we analyzed the relationship between the wall-invasion pattern and the histological phenotype of GBC, using 61 surgically-resected primary gallbladder adenocarcinomas. Histologically, the 61 cases were classified into the biliary ( 44 cases, $72.1 \%$ ), gastric foveolar (13 cases, $21.3 \%$ ), and intestinal (4 cases, 6.6\%) types. Biliary type frequently exhibited MUC1, but less frequently showed MUC2, MUC5AC, and MUC6. The biliary type and MUC1 expression were significantly correlated with DG type wall-invasion pattern $(P=0.020$ and $P<0.001$, respectively). In conclusion, histological phenotype and mucin expression were thought to be indicators of aggressiveness of GBC.
\end{abstract}

Gallbladder carcinoma (GBC) is the most common malignancy of the biliary tract and the fifth most common malignancy of the digestive system. GBC is a relatively uncommon neoplasm in the majority of countries, and its incidence rate shows marked geographic and ethnic variation. Japan and several Latin American countries, such as Chile, Mexico and Bolivia, have the highest incidences of GBC in the world $(11,27)$. In Japan, GBC is responsible for $1.25 \%$ and $3.49 \%$ of cancer death in male and female, respectively. The number of surgically resected cases of GBC has recently increased because of advances in imaging diagnosis and operative procedures. However, GBC is a highly lethal disease since it is usually diagnosed at advance stage (5).

Address correspondence to: Dr Hiroshi Kijima, Department of Pathology and Bioscience, Hirosaki University Graduate School of Medicine, 5 Zaifu-cho, Hirosaki 036-8562, Japan

Tel: +81-172-39-5029, Fax: +81-172-39-5030

E-mail: hkijima@cc.hirosaki-u.ac.jp
The 5-year survival rate of patients with $\mathrm{GBC}$ is $10-30 \%$ despite of surgical resection. Moreover, the majority of patients have frequent recurrences after the surgery, and pursue the chemotherapy or radiotherapy treatment when recurrences happened after the surgery $(2,15)$.

Several previous studies have demonstrated high risks of disease progression, using surgically-resected GBC cases, and histological grade, depth of wall infiltration, and lymph node status have been determined to be clinicopathological prognostic factors $(6$, $22)$. In addition, we have classified the wall-invasion pattern of $\mathrm{GBC}$ into two groups, i.e., infiltrative growth type (IG type) and destructive growth type (DG type) (18-20). The DG type was significantly associated with lymph node metastasis and poor prognosis. In this study, we analyzed the relationship between the wall-invasion pattern and the histological phenotype of GBC, using 61 surgically-resected primary gallbladder adenocarcinomas. 


\section{MATERIALS AND METHODS}

Patient and tissue samples. A total of 61 cases of GBC were retrieved from archival files of Hirosaki University Hospital (Aomori, Japan), in the period between 2000 from 2012. We examined 61 patients (18 males and 43 females) with a mean age of 68.9 years (range, 44-92 years) who received curative resection and lymph node resection. GBC were tumors invading beyond the gallbladder wall, i.e. tumors greater than or equal to pT2, according to the TNM classification (24). The present study followed the principles of the World Medical Association Declaration of Helsinki 1964.

Histopathological and immunohistochemical examinations. The gallbladder tissue specimens for histological analysis were routinely fixed with formalin, embedded in paraffin, thin-sectioned, and stained with hematoxylin and eosin (H\&E). We divided GBC into two wall invasion pattern groups, IG type and DG type. IG type, cancer cells show infiltrative growth in the muscle layer (through the intermuscular space) without muscle layer destruction. DG type, cancer cells invade the subserosal layer with destruction of the muscle layer. The DG type was accompanied by a stromal desmoplastic reaction characterized by active irregular fibrosis due to tumor invasion. Furthermore, according to the WHO classification (1), histological phenotype of the GBC was divided into three groups as follows: (a) Biliary type: the carcinoma was composed of short or long tubular glands lined by cells that vary in height from cuboidal to tall columnar, superficially resembling biliary epithelium; (b) Gastric foveolar type: the carcinoma was composed of tall columnar cells with basally oriented nuclei and abundant mucincontaining cytoplasm; and (c) Intestinal type: the carcinoma was composed of tubular glands closely resembling those of colonic adenocarcinomas, or consisted of glands lined predominantly with goblet cells, and usually with a variable number of neuroendocrine and Paneth cells. We used a term "metaplastic type" as the "gastric foveolar type plus intestinal type." The degree of venous invasion was classified as: v0, no venous invasion; v1, minimal venous invasion, i.e. 1 or 2 foci of venous invasion in one histological section; v2, moderate venous invasion, i.e. 3 or 4 foci; and v3, severe venous invasion with 5 or more foci. The degree of lymphatic invasion was classified as: ly0, no lymphatic invasion; ly1, mild lymphatic invasion; ly2, moderate lymphatic invasion; and ly3, severe lymphatic invasion. The degree of perineural invasion was classified as: ne0, no perineural invasion; ne1, mild perineural invasion; ne2, moderate perineural invasion; and ne3, severe perineural invasion. The subserosal invasion patterns were classified into three groups, according to Classification of Biliary Tract Carcinoma $(8,20)$, i.e. INF $\alpha$ (Infiltration alpha): the tumor shows expanding growth and a distinct border from the surrounding tissue; INF $\beta$ (Infiltration beta): the growth fashion and invasive pattern are between those of INF $\alpha$ and INF $\gamma$ (Infiltration gamma); and INF $\gamma$ : the tumor shows infiltrating growth and an distinct border from the surrounding tissue.

Immunohistochemistry. Representative histological specimens at the deepest invaded area of the gallbladder carcinoma lesions were selected for immunohistochemistry. Four $\mu \mathrm{m}$ thick paraffin sections were mounted on silane-coated glass slides. Immunohistochemical examination was performed on deparaffinized sections using the standard avidinbiotin-peroxidase complex (ABC) method with an automated immnostainer (BenchMarrk XT; Ventana Medical System, Tucson, AZ, USA). We used MUC1, MUC2, MUC5AC and MUC6 to clarify mucin expression of GBC. The antibodies used are listed in Table 1.

Histological and immunohistochemical evaluation. Two investigators (TT and $\mathrm{KH}$ ) simultaneously assessed the immunohistochemical results without any patient's clinicopathological data. Luminal membranous immunoreactivities of the tumor were judged as positive for MUC1, and cytoplasmic immunoreactivities as positive for MUC2, MUC5AC and MUC6. According to the above immunohistochemical expression of each mucin, the cases were divid-

Table 1 Antibodies for immunohistochemistry

\begin{tabular}{ccccc}
\hline Antigen & Monoclonal/polyclonal & Clone & Dilution & Source \\
\hline MUC1 & Monoclonal, mouse & Ma696 & $1: 50$ & Novocastra \\
MUC2 & Monoclonal, mouse & Ccp59 & $1: 50$ & Novocastra \\
MUC5AC & Monoclonal, mouse & CLH2 & $1: 100$ & Novocastra \\
MUC6 & Monoclonal, mouse & CHL5 & $1: 100$ & Novocastra \\
\hline
\end{tabular}


ed into two groups; a negative group in which < $10 \%$ of tumor cells were stained, and a positive group in which $\geqq 10 \%$ were stained.

Statistics. Univariate statistical analysis was performed using the Pearson's chi-square test or Fisher's exact test. Differences were considered to be significant if the $P$-value was less than 0.05 . All statistical analyses were performed using the R (http://www. r-project.org).

\section{RESULTS}

Wall-invasion pattern and clinicopathological factors of $G B C$

Scheme/microscopic findings of wall-invasion pattern and mucin expression are shown in Fig. 1 and Table 2, respectively. Of the 61 cases of GBC, 32 cases $(52.5 \%)$ were IG type, and 29 cases $(47.5 \%)$ were DG type. All of the DG type GBC showed strong MUC1 expression, while MUC2, MUC5AC, and MUC6 were less frequently detected in the DG
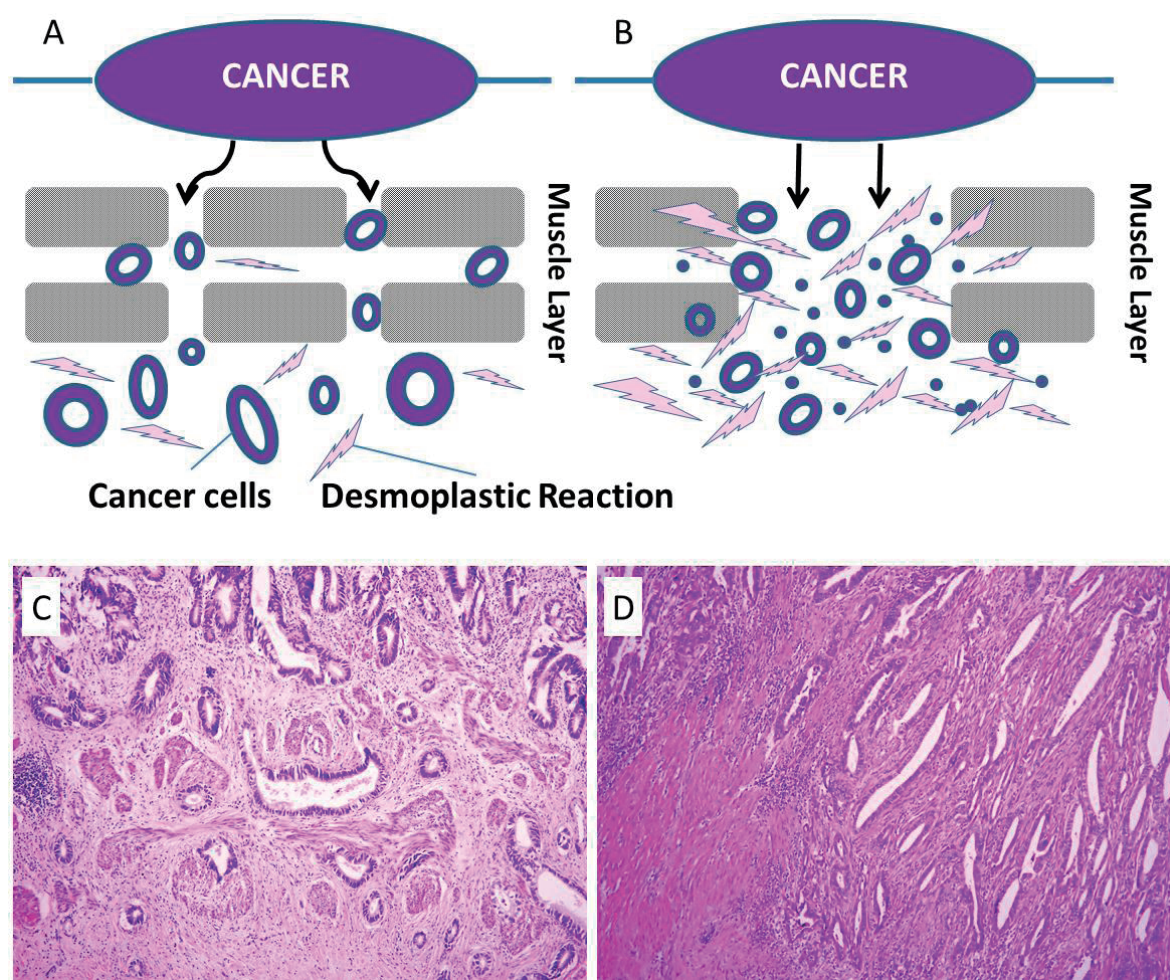

Fig. 1 Scheme and histological findings of advanced GBC invasion pattern. In IG type (A and C), cancer cells show infiltrative growth in the muscle layer (through the intermuscular space) without muscle layer destruction. In DG type (B and D), cancer cells invade the subserosal layer with destruction of the muscle layer. The DG type was accompanied by a stromal desmoplastic reaction which is active and irregular fibrosis due to the tumor invasion.

Table 2 Mucin expression of gallbladder adenocarcinoma

\begin{tabular}{crrcc}
\hline & MUC1 & MUC2 & MUC5AC & MUC6 \\
\hline $\begin{array}{c}\text { GBCs (total } \mathrm{n}=61) \\
\text { Wall-invasion pattern }\end{array}$ & $70.5 \%$ & $14.8 \%$ & $41.0 \%$ & $37.7 \%$ \\
IG type ( $\mathrm{n}=32)$ & $43.8 \%$ & $28.1 \%$ & $62.5 \%$ & $59.4 \%$ \\
DG type (n=29) & $100 \%$ & $0 \%$ & $17.2 \%$ & $13.8 \%$ \\
Histological phenotype & & & & \\
Biliary type (n=44) & $77.3 \%$ & $11.4 \%$ & $27.3 \%$ & $22.7 \%$ \\
Gastric foveolar type (n=13) & $61.5 \%$ & $15.4 \%$ & $84.6 \%$ & $84.6 \%$ \\
Intestinal type (n=4) & $25.0 \%$ & $50.0 \%$ & $50.0 \%$ & $50.0 \%$ \\
\hline
\end{tabular}

GBCs: gallbladder carcinomas, IG type: infiltrative growth type, DG type: destructive growth type. 
type. The IG type GBC frequently expressed MUC1 (43.8\%), MUC2 (28.1\%), MUC5AC (62.5\%), and MUC6 (59.4\%), but not in all cases. The results of univariate analysis between wall-invasion pattern and clinicopathological factors are summarized in Table 3. Age, gender and histological differentiation exhibited no significant differences. The DG pattern significantly increased lymphatic invasion, venous invasion, lymph node metastasis and neural invasion. The wall-invasion pattern was also associated with spread pattern and histological phenotype.

Histological phenotype and clinicopathological factors of $G B C$

Representative findings of histological phenotype are demonstrated in Fig. 2. Of the 61 cases of GBC, 44 cases $(72.1 \%)$ were biliary type, and 17 cases (27.9\%) were metaplastic type; i.e. 13 (21.3\%) were gastric foveolar type, and $4(6.6 \%)$ were intestinal type. Histological phenotype and mucin expression of GBC are summarized in Table 2 and in Fig. 3. The biliary type GBC frequently showed strong MUC1 expression $(77.3 \%, 34 / 44)$, while MUC2, MUC5AC, and MUC6 were less frequently detected in the biliary type. The gastric foveolar type frequently expressed MUC1 $(61.5 \%, 8 / 13)$ as well as MUC5AC and MUC6 (84.6\%, 11/13; and 84.6\%, $11 / 13$, respectively). The results of univariate analysis between histological phenotype pattern and clinicopathological factors are summarized in Table 4. The biliary type was significantly correlated with the DG type wall-invasion, but was not statistically associated with the other clinicopathological factors.

\section{DISCUSSION}

In the present study, we investigated the wall-invasion pattern and histological phenotype of GBC,

Table 3 The wall-invasion pattern and clinicopathological features of gallbladder adenocarcinoma

\begin{tabular}{|c|c|c|c|c|c|}
\hline \multirow{2}{*}{$\begin{array}{l}\text { Clinicopathological } \\
\text { features }\end{array}$} & \multirow{2}{*}{$\begin{array}{l}\text { Number of } \\
\text { patients }\end{array}$} & \multicolumn{2}{|c|}{ Wall-invasion pattern } & \multirow{2}{*}{$\begin{array}{l}\text { Rate of DG } \\
\text { pattern }(\%)\end{array}$} & \multirow{2}{*}{ P-value } \\
\hline & & IG & DG & & \\
\hline Age & & & & & 0.053 \\
\hline$<65$ & 18 & 6 & 12 & 66.7 & \\
\hline$\geqq 65$ & 43 & 26 & 17 & 39.5 & \\
\hline Gender & & & & & 0.417 \\
\hline Male & 18 & 8 & 10 & 55.6 & \\
\hline Female & 43 & 24 & 19 & 44.2 & \\
\hline Histological differentiation & & & & & 0.095 \\
\hline well, mod & 44 & 26 & 18 & 40.9 & \\
\hline por, others & 17 & 6 & 11 & 64.7 & \\
\hline Lymphatic invasion & & & & & $0.003^{*}$ \\
\hline ly 0,1 & 31 & 22 & 9 & 29.0 & \\
\hline $\operatorname{ly} 2,3$ & 30 & 10 & 20 & 66.7 & \\
\hline Venous invasion & & & & & $0.032 *$ \\
\hline v0, 1 & 36 & 23 & 13 & 36.1 & \\
\hline v2, 3 & 25 & 9 & 16 & 64.0 & \\
\hline Neural invasion & & & & & $0.016^{*}$ \\
\hline ne 0,1 & 37 & 24 & 13 & 35.1 & \\
\hline ne 2,3 & 24 & 8 & 16 & 66.7 & \\
\hline LN metastasis & & & & & $0.003 *$ \\
\hline+ & 30 & 10 & 20 & 66.7 & \\
\hline- & 31 & 22 & 9 & 29.0 & \\
\hline Spread pattern & & & & & $0.003^{*}$ \\
\hline $\mathrm{INF} \alpha, \beta$ & 35 & 24 & 11 & 31.4 & \\
\hline INF $\gamma$ & 26 & 8 & 18 & 69.2 & \\
\hline Phenotype & & & & & $0.020^{*}$ \\
\hline Biliary type & 44 & 19 & 25 & 56.8 & \\
\hline Metaplastic type & 17 & 13 & 4 & 23.5 & \\
\hline
\end{tabular}

IG: infiltrative growth type, DG: destructive growth type, well: well differentiated adenocarcinoma, mod: moderately differentiated adenocarcinoma, por: poorly differentiated adenocarcinoma, ly: lymphatic invasion, v: venous invasion, ne: neural invasion, INF $\alpha, \beta$ and $\gamma$ : the mode of subserosal infiltration. *Statistically significant: $P<0.05$. 

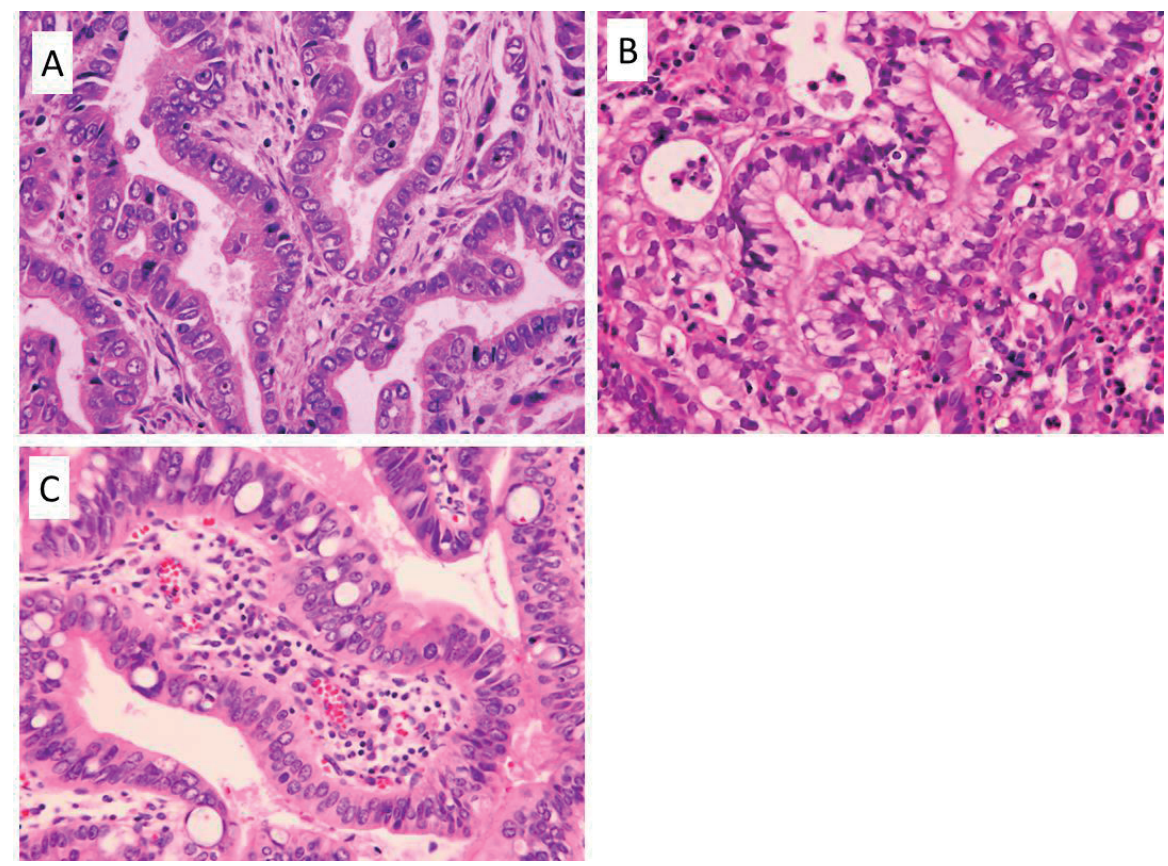

Fig. 2 Three morphological subtypes of GBC (hematoxylin and eosin). (A) Biliary type: tumor was composed of short or long tubular glands lined by cells that vary in height from cuboidal to tall columnar, superficially resembling biliary epithelium. (B) Gastric foveolar type: tumor was composed of tall columnar cells with basally oriented nuclei and abundant mucincontaining cytoplasm, and (C) Intestinal type: tumor was composed of tubular glands closely resembling those of colonic adenocarcinomas, and consisted of glands lined predominantly with goblet cells.

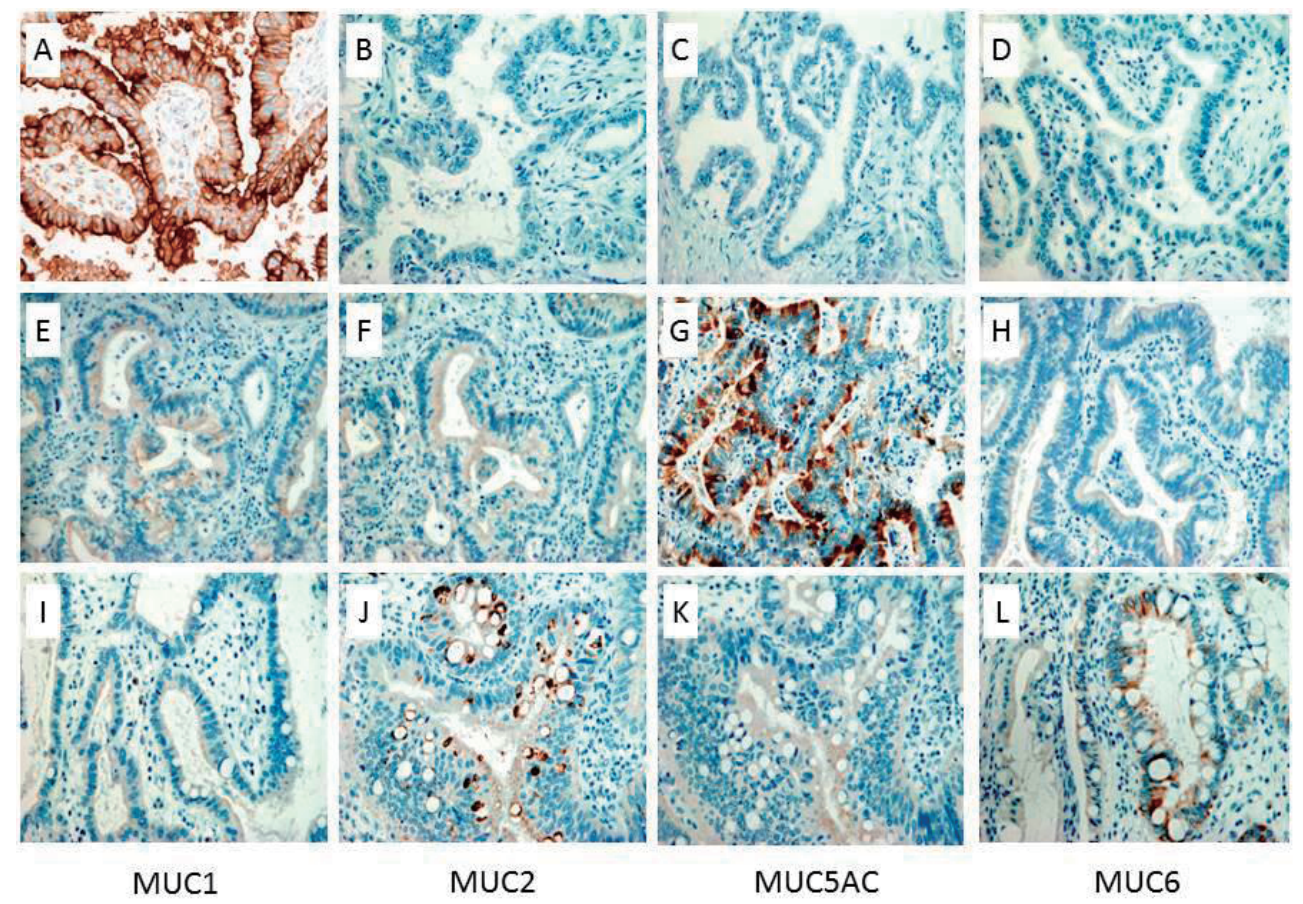

Fig. 3 Representative mucin expression images of GBCs. A case of biliary type (A)-(D). A case of gastric foveolar type $(E)-(H)$ and a case of intestinal type (I)-(L). Immunochemical staining of MUC1 (A, E, I), MUC2 (B, F, J), MUC5AC (C, G, $\mathrm{K})$, and MUC6 (D, H, L). Biliary type tumor (A)-(D) shows MUC1 positive, MUC2, MUC5AC and MUC6 negative. Gastric foveolar type tumor $(\mathrm{E})-(\mathrm{H})$ shows MUC5AC positive, MUC1, MUC2 and MUC6 negative. Intestinal type tumor (I)-(L) shows MUC2 and MUC6 positive, MUC1 and MUC5AC negative. 
Table 4 Histological phenotype and clinicopathological features of adenocarcinoma

\begin{tabular}{|c|c|c|c|c|}
\hline \multirow{2}{*}{$\begin{array}{c}\text { Clinicopathological } \\
\text { features }\end{array}$} & \multirow{2}{*}{$\begin{array}{l}\text { Number of } \\
\text { patients }\end{array}$} & \multicolumn{2}{|c|}{ Histological phenotype } & \multirow{2}{*}{ P-value } \\
\hline & & Biliary & Metaplastic & \\
\hline Age & & & & 0.524 \\
\hline$<65$ & 18 & 14 & 4 & \\
\hline$\geqq 65$ & 43 & 30 & 13 & \\
\hline Gender & & & & 0.207 \\
\hline Male & 18 & 15 & 3 & \\
\hline Female & 43 & 29 & 14 & \\
\hline Histological differentiation & & & & 0.268 \\
\hline well, mod & 44 & 30 & 14 & \\
\hline por, others & 17 & 14 & 3 & \\
\hline Lymphatic invasion & & & & 0.715 \\
\hline ly 0,1 & 31 & 23 & 8 & \\
\hline ly 2,3 & 30 & 21 & 9 & \\
\hline Venous invasion & & & & 0.574 \\
\hline $\mathrm{v} 0,1$ & 36 & 25 & 11 & \\
\hline v2, 3 & 25 & 19 & 6 & \\
\hline Neural invasion & & & & 0.177 \\
\hline ne 0,1 & 37 & 29 & 8 & \\
\hline ne2, 3 & 24 & 15 & 9 & \\
\hline Spread pattern & & & & 0.195 \\
\hline $\mathrm{INF} \alpha, \beta$ & 35 & 23 & 12 & \\
\hline $\mathrm{INF} \gamma$ & 26 & 21 & 5 & \\
\hline $\mathrm{LN}$ metastasis & & & & 0.715 \\
\hline+ & 30 & 21 & 9 & \\
\hline- & 31 & 23 & 8 & \\
\hline Wall-invasion pattern & & & & $0.020^{*}$ \\
\hline IG & 32 & 19 & 13 & \\
\hline DG & 29 & 25 & 4 & \\
\hline
\end{tabular}

well: well differentiated adenocarcinoma, mod: moderately differentiated adenocarcinoma, por: poorly differentiated adenocarcinoma, ly: lymphatic invasion, v: venous invasion, ne: neural invasion, INF $\alpha, \beta$ and $\gamma$ : the mode of subserosal infiltration, IG: infiltrative growth type, DG: destructive growth type. *Statistically significant: $P<0.05$.

using 61 surgically-resected primary gallbladder adenocarcinomas. GBC cases were classified into the biliary, gastric foveolar, and intestinal types. The biliary type and MUC1 expression were significantly correlated with the DG type wall-invasion pattern. This is the first report to demonstrate biliary type/MUC1 expression as indicators of aggressiveness of GBC.

Several studies have reported prognostic significance of histological phenotype and mucin expression in $\operatorname{GBC}(3,9,10,21,25)$. However, the correlation between the histological phenotype/mucin expression and wall-invasion pattern has not yet clarified. Mucin is a heterogeneous group of high molecular weight glycoproteins with many carbohydrate side chains. The mucin shares the common features of having an extensive tandem repeat region, and a peptide domain containing a high per- centage of serine and threonine $(4,12,16)$. Recently, 19 human mucin genes have been assigned to the MUC gene family. MUC1, located in 1q21-23, is a transmembrane mucin and is expressed in pancreatobiliary cancer, and may function as an anti-adhesion molecule that inhibits homotypical cell aggregation and adhesion to the extracellular matrix, promoting cell invasion (14). MUC2, MUC5AC and MUC6, which are clustered within the $11 \mathrm{p} 15$ locus, are expressed in intestinal goblet cells, gastric foveolar cells and pyloric gland cells $(23,26)$, respectively. Histological phenotype of GBC is thought to modulate the biological behavior of carcinoma cells such as tumor progression.

We previously clarified that the DG type wallinvasion pattern was significantly associated with poor differentiation, aggressive infiltration, and decreased postoperative survival in terms of its histo- 
logical differentiation, lymphatic invasion, venous invasion, lymph node status, neural invasion, and mode of subserosal infiltration (18-20). In present study, both histological phenotype and MUCs expression were significantly correlated with wall-invasion pattern of GBC. Several studies have demonstrated that different histological phenotypes expressed different mucins; i.e. mucin expression on carcinoma cells representing a metaplastic phenotype was qualitatively and quantitatively different from those of non-metaplastic phenotype $(7,13,17)$. Therefore, the biliary type and MUC1 expression were thought to be significantly correlated with DG type, i.e. the more aggressive wall-invasion pattern. In conclusion, histological phenotype and mucin expression were thought to be indicators of aggressiveness of GBC.

\section{Acknowledgements}

This study was supported by Grants-in-aid for Science from the Ministry of Education, Culture, Sports, Science, and Technology in Japan; and a Grant for Hirosaki University Institutional Research.

\section{REFERENCES}

1. Albores-Saavedra J, Adsay NV, Crawford JM, Klimstra DS and Klöppel G (2010) Carcinoma of the gallbladder and extrahepatic bile ducts. In: World Health Organization of Classification of Tumors of the Digestive System 4th Edition (Bosman FT, Carneiro F, Hruban RH and Theise ND, eds), pp264-278, International Agency for Research on Cancer, Lyon.

2. Balachandran P, Agarwal S, Krishnani N, Pandey CM, Kumar A, Sikora SS, Saxena R and Kapoor VK (2006) Predictors of long-term survival in patients with gallbladder cancer. J Gastrointest Surg 10, 848-854.

3. Chang HJ, Kim SW, Lee BL, Hong EK and Kim WH (2004) Phenotypic alterations of mucins and cytokeratins during gallbladder carcinogenesis. Pathol Int 54, 576-584.

4. Dekker J, Rossen JW, Buller HA and Einerhand AW (2002) The MUC family: an obituary. Trends Biochem Sci 27, 126131.

5. Gourgiotis S, Kocher HM, Solaini L, Yarollahi A, Tsiambas E and Salemis NS (2008) Gallbladder cancer. Am J Surg 196, 252-264.

6. Hara S, Kijima H, Okada K and Igarashi Y (2010) Invasive micropapillary variant of the gallbladder adenocarcinoma and its aggressive potential for lymph node metastasis. Biomed Res (Tokyo) 31, 89-95.

7. Irimura T, Matsushita Y, Hoff SD, Yamori T, Nakamori S, Frazier ML, Giacco GG, Cleary KR and Ota DM (1991) Ectopic expression of mucins in colorectal cancer metastasis. Semin Cancer Biol 2, 129-139.

8. Japanese Society of Biliary Surgery (2004) Classification of Biliary Tract Carcinoma, 2nd English ed., pp33-50, Kanehara and Co., Ltd., Tokyo.

9. Kashiwagi H, Kijima H, Dowaki S, Ohtani Y, Tobita K,
Yamazaki H, Nakamura M, Ueyama Y, Tanaka M, Inokuchi S and Makuuchi H (2001) MUC1 and MUC2 expression in human gallbladder carcinoma: a clinicopathological study and relationship with prognosis. Oncol Rep 8, 485-489.

10. Kawamoto T, Shoda J, Irimura T, Miyahara N, Furukawa M, Ueda T, Asano T, Kano M, Koike N, Fukao K, Tanaka N and Todoroki T (2001) Expression of MUC1 mucins in the subserosal layer correlates with postsurgical prognosis of pathological tumor stage 2 carcinoma of the gallbladder. Clin Cancer Res 7, 1333-1342.

11. Kayahara M and Nagakawa T (2007) Recent trends of gallbladder cancer in Japan: an analysis of 4,770 patients. Cancer 110, 572-580.

12. Kim YS, Gum JR, Jr., Crawley SC, Deng G and Ho JJ (1999) Mucin gene and antigen expression in biliopancreatic carcinogenesis. Ann Oncol 10 Suppl 4, 51-55.

13. Kudo Y, Morohashi S, Takasugi K, Tsutsumi S, Ogasawara H, Hanabata N, Yoshimura T, Sato F, Fukuda S and Kijima H (2011) Histopathological phenotypes of early gastric cancer and its background mucosa. Biomed Res (Tokyo) 32, 127-134.

14. Ligtenberg MJ, Buijs F, Vos HL and Hilkens J (1992) Suppression of cellular aggregation by high levels of episialin. Cancer Res 52, 2318-2324.

15. Malka D, Boige V, Dromain C, Debaere T, Pocard M and Ducreux M (2004) Biliary tract neoplasms: update 2003. Curr Opin Oncol 16, 364-371.

16. Moniaux N, Escande F, Porchet N, Aubert JP and Batra SK (2001) Structural organization and classification of the human mucin genes. Front Biosci 6, D1192-1206.

17. Nicolson GL (1982) Cancer metastasis. Organ colonization and the cell-surface properties of malignant cells. Biochim Biophys Acta 695, 113-176.

18. Okada K, Kijima H, Imaizumi T, Hirabayashi K, Matsuyama M, Yazawa N, Dowaki S, Tobita K, Ohtani Y, Tanaka M, Inokuchi S and Makuuchi H (2012) Clinical significance of wall invasion pattern of subserosa-invasive gallbladder carcinoma. Oncol Rep 28, 1531-1536.

19. Okada K, Kijima H, Imaizumi T, Hirabayashi K, Matsuyama M, Yazawa N, Oida Y, Dowaki S, Tobita K, Ohtani Y, Tanaka M, Inokuchi S and Makuuchi H (2009) Stromal laminin5 gamma2 chain expression is associated with the wall-invasion pattern of gallbladder adenocarcinoma. Biomed Res (Tokyo) 30, 53-62.

20. Okada K, Kijima H, Imaizumi T, Hirabayashi K, Matsuyama M, Yazawa N, Oida Y, Dowaki S, Tobita K, Ohtani Y, Tanaka M, Inokuchi S and Makuuchi H (2009) Wall-invasion pattern correlates with survival of patients with gallbladder adenocarcinoma. Anticancer Res 29, 685-691.

21. Park SY, Roh SJ, Kim YN, Kim SZ, Park HS, Jang KY, Chung MJ, Kang MJ, Lee DG and Moon WS (2009) Expression of MUC1, MUC2, MUC5AC and MUC6 in cholangiocarcinoma: prognostic impact. Oncol Rep 22, 649-657.

22. Roa I, de Aretxabala X, Araya JC, Villaseca M, Roa J, Gilda IT, Burgos L and Munoz S (2002) Morphological prognostic elements in gallbladder cancer. Rev Med Chil 130, 387-395.

23. Sasaki M, Yamato T, Nakanuma Y, Ho SB and Kim YS (1999) Expression of MUC2, MUC5AC and MUC6 apomucins in carcinoma, dysplasia and non-dysplastic epithelia of the gallbladder. Pathol Int 49, 38-44.

24. Sobin LH, Gospodarowicz MK and Wittekind CH (2009) TNM Classification of Malignant Tumours (UICC), 7th Edition. Wiler-Liss, New York.

25. Yamamoto M, Nakajo S and Tahara E (1989) Carcinoma of the gallbladder: the correlation between histogenesis and 
prognosis. Virchows Arch A Pathol Anat Histopathol 414, 83-90.

26. Yamato T, Sasaki M, Watanabe Y and Nakanuma Y (1999) Expression of MUC1 and MUC2 mucin core proteins and their messenger RNA in gall bladder carcinoma: an immunohistochemical and in situ hybridization study. J Pathol 188,
30-37.

27. Yokoyama N, Hitomi J, Watanabe H, Ajioka Y, Pruyas M, Serra I, Shirai Y and Hatakeyama K (1998) Mutations of p53 in gallbladder carcinomas in high-incidence areas of Japan and Chile. Cancer Epidemiol Biomarkers Prev 7, 297-301. 